\title{
Evaluating the Impact of a Blood Pressure Remote Telemonitoring Program (BP Connect)
}

Emily de Redon ${ }^{1}$, BA; Ramya Palacholla ${ }^{1,2,3}$, MD, MPH; Sara Golas ${ }^{1}$, MA; Nina Schussler ${ }^{1}$, BA; Lauren Cortese ${ }^{1}$, BA; Sharon Odametey ${ }^{1}$, BA, MPH; Stephen Agboola ${ }^{1,2,3}$, MD, MPH; Joseph Kvedar ${ }^{1,2,3}$, MD; Kamal Jethwani ${ }^{1,2,3}$, $\mathrm{MD}, \mathrm{MPH}$

\author{
${ }^{1}$ Partners Connected Health, Boston, MA, United States \\ ${ }^{2}$ Harvard Medical School, Boston, MA, United States \\ ${ }^{3}$ Massachusetts General Hospital, Boston, MA, United States
}

Corresponding Author:

Emily de Redon, BA

Partners Connected Health

25 New Chardon Street

Boston, MA,

United States

Phone: 17242155

Email: ederedon@partners.org

\section{Abstract}

Background: The prevalence of hypertension is around 30-45\% among the general population. Hypertension contributes to 1 out of 7 deaths in the United States and approximately $70 \%$ of persons who have a first heart attack or stroke. Timely treatment and optimal management of hypertension is associated with substantial reductions in stroke incidence (35-40\%), myocardial infarction (20-25\%), and heart failure (>50\%). Additionally, remote monitoring with active intervention by medical professionals (telemonitoring) improves drug compliance and increase the target blood pressure (BP) achievement rate.

Objective: BP Connect is a remote monitoring program to augment care and disease self-management in hypertensive patients. The program aims to engage patients in self-care by providing a secure web-based platform to record and track their BP. In addition, care providers can view the uploaded data, thereby, ensuring the continuum of care beyond the hospital setting.

Methods: A total of 288 adult patients diagnosed with hypertension (baseline blood pressure of $\geq 140 \mathrm{~mm} \mathrm{Hg}$ systolic or $\geq 90$ $\mathrm{mm} \mathrm{Hg}$ diastolic) were recruited from primary care and specialty clinics within the Partners Healthcare network of hospitals (Faulkner, Renal, Endocrinology and Cardiology departments at Brigham and Women's Hospital and Massachusetts General Hospitals Women's Health Association). The primary outcome was the impact of BP Connect on blood pressure as measured by the change in the proportion of patients with controlled BP (ie, $<140 / 90 \mathrm{~mm} \mathrm{Hg}$ ) from the initial visit to the 3-month clinic visit. Secondary outcomes included the change in systolic blood pressure (SBP) and diastolic blood pressure (DBP) at close-out.

Results: Among the 149 patients from primary care clinics who completed the program to-date $48.0 \%$ of patients had uncontrolled $\mathrm{BP}(>140 / 90 \mathrm{~mm} \mathrm{Hg})$ vs $64.0 \%$ at baseline $(P=.01)$. At 3 months, there was a significant decline in the mean SBP $(-10.1, P<.01)$ and the mean DBP $(-4.3, P<.01)$. Among the 139 patients from specialty clinics who completed this program, BP was controlled at 3 months in $43.2 \%$ of patients compared with $11.2 \%$ patients at baseline $(P=.001)$. A significant decrease in the mean SBP $(-5.2, P<.001)$ and in the mean DBP $(-2.5, P=.01)$ were also noted.

Conclusions: Overall, the BP Connect program shows potential to improve clinical outcomes in hypertensive patients. The program provides an opportunity for participants to track their BP measurements on a web platform to foster patient participation in their own disease management that may improve patient outcomes and decrease burden on the care providers.

(iproc 2017;3(1):e20) doi: 10.2196/iproc.8455

\section{Multimedia Appendix 1}

Full poster. 
Edited by T Hale; this is a non-peer-reviewed article. Submitted 13.07.17; accepted 24.08.17; published 22.09.17.

Please cite as:

de Redon E, Palacholla R, Golas S, Schussler N, Cortese L, Odametey S, Agboola S, Kvedar J, Jethwani K

Evaluating the Impact of a Blood Pressure Remote Telemonitoring Program (BP Connect)

iproc 2017;3(1):e20

URL: http://www.iproc.org/2017/1/e20/

doi: 10.2196/iproc.8455

PMID:

CEmily de Redon, Ramya Palacholla, Sara Golas, Nina Schussler, Lauren Cortese, Sharon Odametey, Stephen Agboola, Joseph Kvedar, Kamal Jethwani. Originally published in Iproceedings (http://www.iproc.org), 22.09.2017. This is an open-access article distributed under the terms of the Creative Commons Attribution License (https://creativecommons.org/licenses/by/4.0/), which permits unrestricted use, distribution, and reproduction in any medium, provided the original work, first published in Iproceedings, is properly cited. The complete bibliographic information, a link to the original publication on http://www.iproc.org/, as well as this copyright and license information must be included. 